\title{
A Case of Migration of Pipeline Embolization Device Causing Rupture during Treatment of an Unruptured Vertebral Artery Dissecting Aneurysm
}

\author{
Sung Ho Kim, Dong Kyu Yeo, Gwang Soo Lee \\ Department of Neurosurgery, Soonchunhyang University Gumi Hospital, Gumi, Korea
}

\begin{abstract}
Endoluminal reconstruction of an intracranial aneurysm using flow-diverting devices, such as the pipeline embolization device (PED), is a new treatment modality with good clinical outcomes. The device was originally indicated for challenging cases, such as wide-necked large or giant aneurysms, and is gaining popularity as a reliable treatment for nearly all intracranial aneurysms. The overall complication rate of flow-diverting devices use is $17.0 \%$, including occlusion of side-branching or perforating arteries, rerupture of the aneurysm, in-stent thrombosis, and, rarely, stent migration. We report a rare complication of the PED: delayed migration of the PED after successful stent implantation during treatment of an unruptured vertebral artery dissecting aneurysm, which resulted in rupture of the aneurysm. Further, we discuss technical steps that can be taken to prevent this potential complication.
\end{abstract}

Keywords: Flow diverting device; Migration; Aneurysm rupture; Complication; Case report

\section{INTRODUCTION}

Flow-diverting devices (FDDs), such as the pipeline embolization device (PED), are a new generation of stents introduced recently that were originally indicated for challenging cases of widenecked or fusiform aneurysms from the petrous to the clinoid segment of the internal carotid artery. However, they are expanding indications with good clinical outcome of high success rate of $50 \%-76 \%$ at 6 months and $86 \%$ at 1 year and low mortality and morbidity rates of $0 \%-8 \%$ and $3.9 \%-15 \%$, respectively [1]. Because FDDs use is expected to increase, it is important to know about the complications that can be caused by FDDs to help prevent them. We report a rare complication of a PED: delayed migration of the PED after successful stent implantation during treatment of an unruptured vertebral artery dissecting aneurysm (VADA) that resulted in rupture of the aneurysm.

\section{CASE REPORT}

A 19-year-old male visited Soonchunhyang University Gumi Hospital with a headache. The patient did not have any other med- ical history, and brain magnetic resonance imaging (MRI) and magnetic resonance angiography showed a large VADA on the right vertebral artery (VA) compressing the medulla oblongata (Fig. 1). Digital subtraction angiography (DSA) indicated a VADA on nearly the entire length of the right V4 segment just proximal to the posterior inferior cerebellar artery with bleb formation and a retrograde flow from the left VA to the VADA (Fig. 2). The VADA was unruptured, but there was a mass effect on the brainstem presenting as a headache, and because of the bleb, it seemed to have a risk of rupture; therefore, reconstructive treatment using a PED was planned. The diameters of the VA distal and proximal to the VADA segment were $3.71 \mathrm{~mm}$ and $3.08 \mathrm{~mm}$, respectively, and the length of the dissecting segment was $27.33 \mathrm{~mm}$. We tried not to cover the basilar artery (BA) as much as possible, to avoid perforator occlusion, and $4.25 \times 30 \mathrm{~mm}$ PED (ev3; Medtronic-Covidien Neurovascular Inc., Irvine, CA, USA) was deployed (Fig. 3A). Upon deployment of the stent on the dissecting segment, stable wall apposition of the stent by pushing the device was difficult due to the large diameter of the dissection; therefore, microcatheter unsheathing was done for a long distance since the dissecting segment was long. Final angiogram showed pronounced stasis of
Correspondence to: Sung Ho Kim

Department of Neurosurgery, Soonchunhyang University Gumi Hospital, 179 1(il)gongdan-ro, Gumi 39371, Korea

Tel: +82-54-486-9130, Fax: +82-54-468-9075, E-mail: 68512@schmc.ac.kr

Received: Nov. 13, 2021 / Accepted after revision: Dec. 9, 2021
(C) 2021 Soonchunhyang Medical Research Institute

This is an Open Access article distributed under the terms of the Creative Commons Attribution Non-Commercial License (https://creativecommons.org/licenses/by-nc/4.0/) 

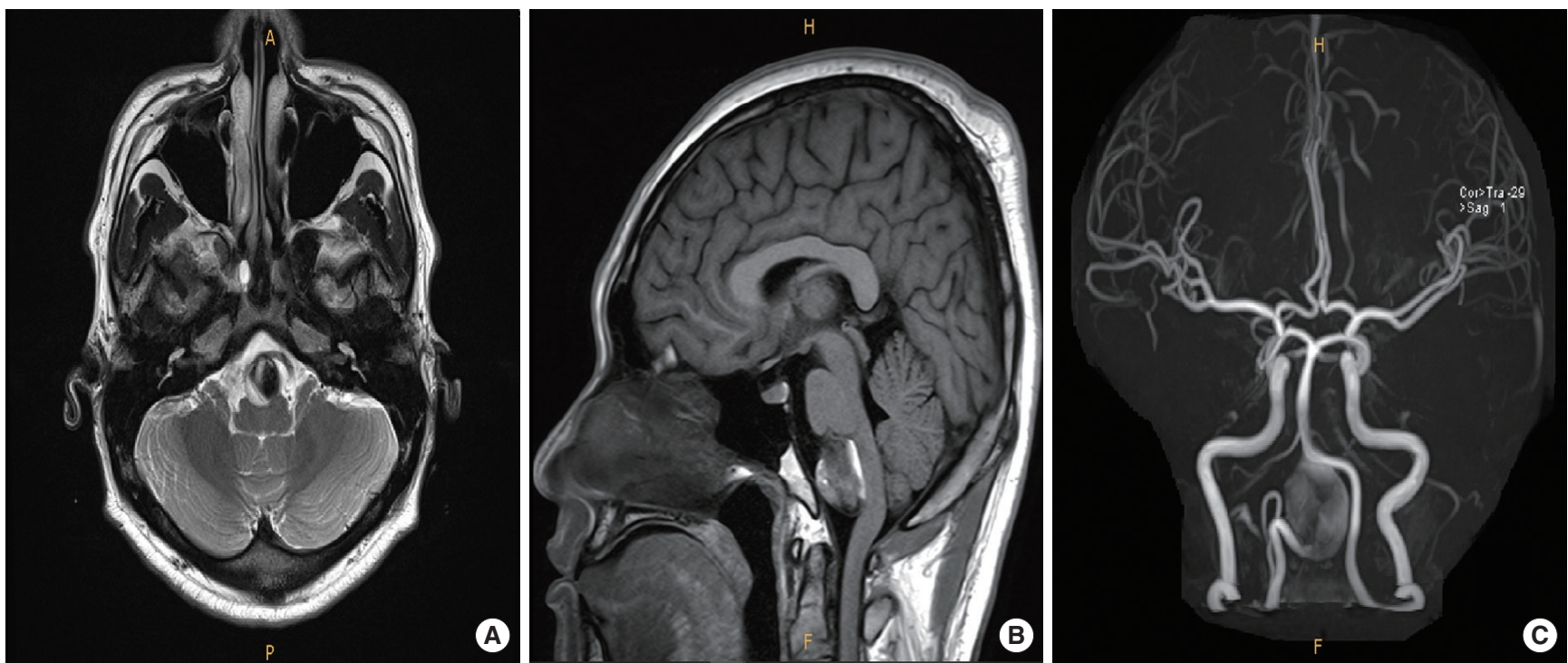

Fig. 1. (A-C) Brain magnetic resonance imaging and magnetic resonance angiography show a huge vertebral artery dissecting aneurysm on the right vertebral artery compressing the medulla oblongata.
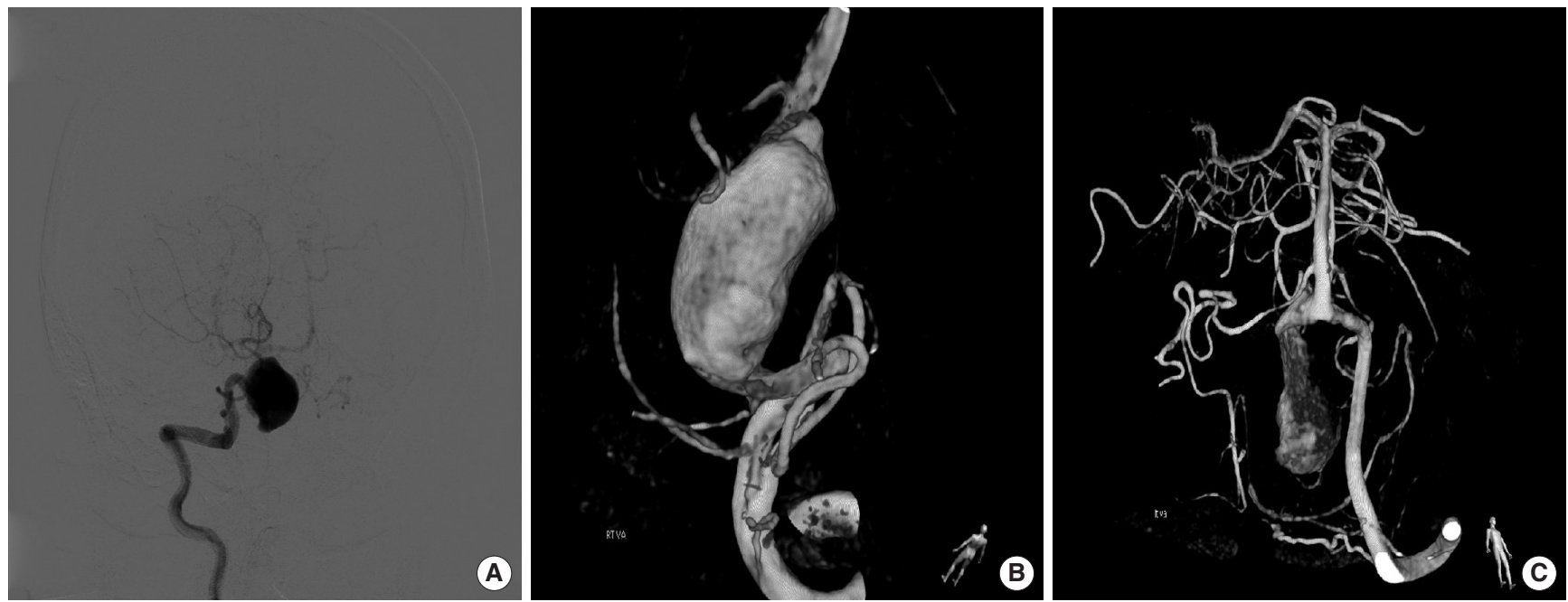

Fig. 2. (A-C) Digital subtraction angiogram and three-dimensional angiographic reconstruction demonstrate a vertebral artery dissecting aneurysm (VADA) along almost the entire length of right $\mathrm{V} 4$ segment just proximal to the posterior inferior cerebellar artery, including a bleb and retrograde flow from the left vertebral artery to the VADA.

contrast, contrast was visualized until venous phase (Fig. 3B). The patient was discharged uneventfully but returned, presenting with a severe headache on postoperative day 4 . Brain computed tomography and DSA showed intraventricular and subarachnoid hemorrhage and indicated the PED we had implanted had migrated proximally due to shortening (Fig. 4). Covering the VADA with another PED in a telescopic fashion was an option, but we did not want to take risk of rebleeding; instead, parent artery occlusion with coils was planned since the contralateral VA was intact. We wanted tight coil-packing on the right VA, but there was a risk of occlusion of the BA during coil-packing because the VADA was near the vertebrobasilar junction; therefore, we decided to implant a stent along the BA to the left VA. To protect the BA from the coils with confidence, and since there was retrograde flow from the left VA to the VADA, the PED was selected as the stent (Fig. 5). During the procedure, there were no special events, but the patient developed abducens nerve palsy on the left side afterwards. Brain MRI showed no additional abnormalities besides the initial hemor- 

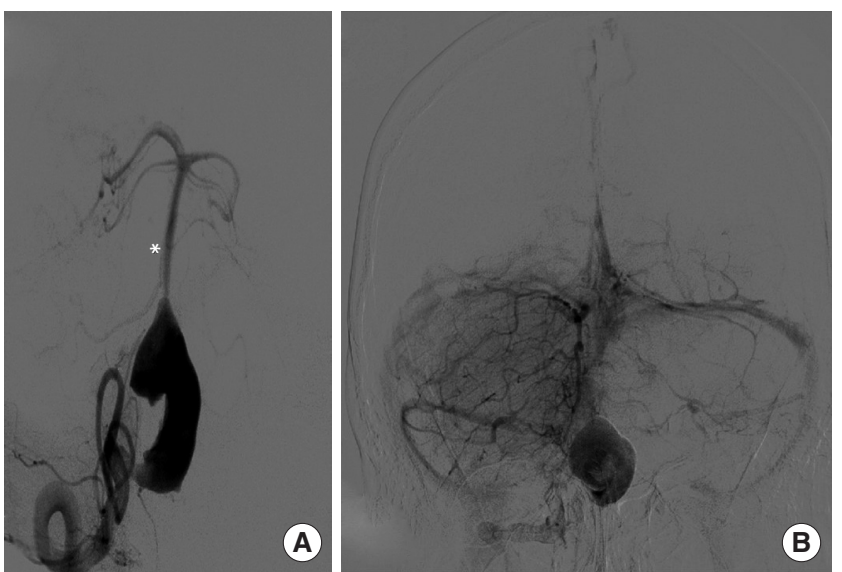

Fig. 3. (A) A $4.25 \times 30 \mathrm{~mm}$ pipeline embolization device (PED) is deployed to cover the area from the right vertebral artery to the basilar artery and the distal end of the stent $\left(^{*}\right)$ is at the proximal basilar trunk. (B) Final digital subtraction angiogram shows a pronounced stasis of contrast, contrast visualizing until venous phase.
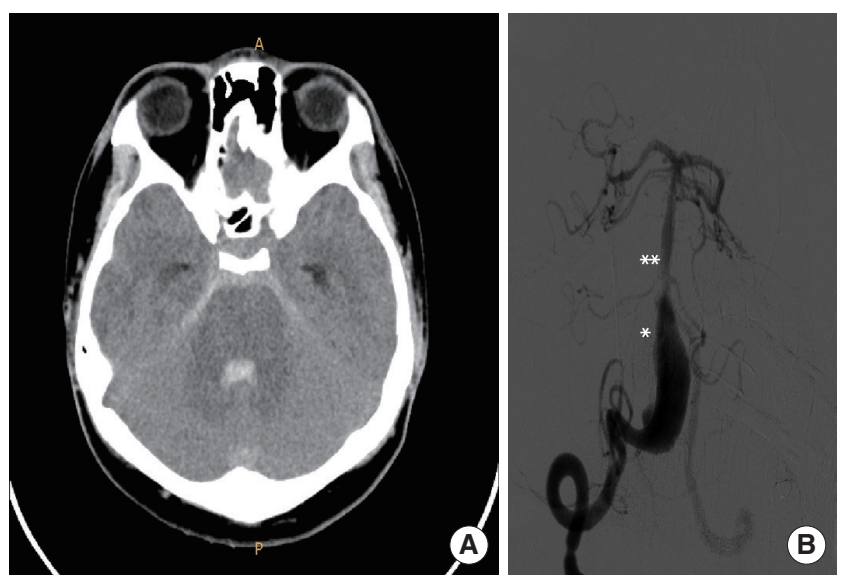

Fig. 4. (A) Brain computed tomography shows intraventricular and subarachnoid hemorrhages. (B) The pipeline embolization device we implanted has migrated proximally due to shortening and the previous distal end of the stent $\left({ }^{* *}\right)$ was on the proximal basilar trunk, but it has migrated into the dissecting segment $\left({ }^{*}\right)$.

rhage. The reason for abducens nerve palsy was not apparent, but the mass effect caused by increased intracranial pressure was assumed. The headache subsided, and the patient discharged with left abducens palsy, which improved over time.

The patient provided written informed consent for the publication of clinical details and images.

\section{DISCUSSION}

Endoluminal reconstruction of an intracranial aneurysm using FDDs is a new treatment modality with good clinical outcomes.

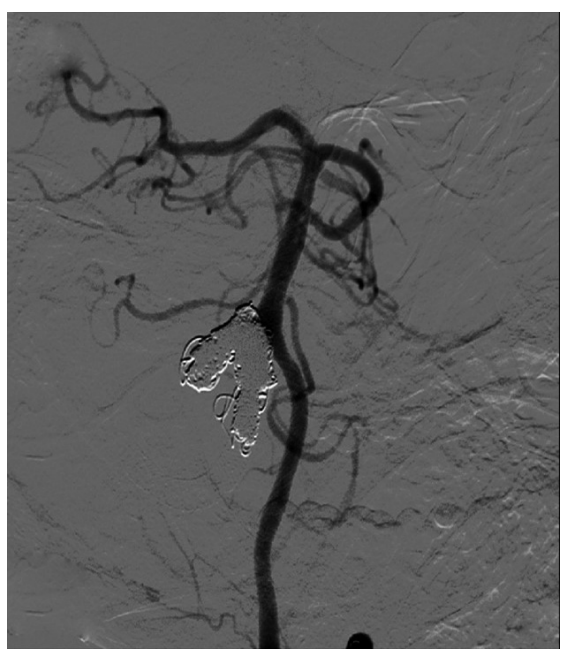

Fig. 5. Occlusion of the parent artery with coils is done at the right vertebral artery, and the pipeline embolization device is implanted from the left vertebral artery to the basilar artery.

Originally indicated for challenging cases, such as wide-necked large or giant aneurysms, it is gaining popularity as a reliable treatment for almost all intracranial aneurysms [2].

FDDs are stents designed to provide greater metal coverage and decreased porosity to induce flow diversion to occlude aneurysms when placed in the parent artery. They provide a redirection of blood flow to create stagnation of the blood in the aneurysm, creating intra-aneurysmal thrombosis, while offering good support for development of neointima.

The overall complication rate of FDDs use is $17.0 \%$, including occlusion of side-branching or perforating arteries, re-rupture of aneurysms, in-stent thrombosis, and, rarely, stent migration [3].

This case report illustrates a complication of delayed stent migration after successful stent implantation which resulted in rupture of the dissecting aneurysm which could have been fatal.

There are several proposed mechanisms of stent migration. First, when there is a significant difference between the luminal diameters of outflow and inflow vessels, a squeezing force can occur toward the larger-diameter side, producing the so-called "watermelon seed effect," which causes stent migration [4]. Also, excessive dragging and stretching of the stent during deployment may induce foreshortening of the stent, namely the "accordion effect," which results in migration. Finally, there is an inherent factor that makes FDDs more vulnerable to spontaneous migration than other types of stents. FDDs have a specific low-porosity and closed-cell design, which facilitates the transmission of a force exerted at one end of the device to the other end, making migration 
easier [3].

In most cases, migration of FDD is silent, but there are reports of aneurysm growth and rupture by hemodynamic changes evoked by FDD migration $[1,3,5,6]$. The rupture of the VADA in this case was thought to be due to the migrated stent, which introduced the bloodstream to the dissected segment, resulting in rupture. Therefore, prevention of FDD migration is critical. Careful selection of size and length and use of a deployment technique that allows stable wall apposition of the stent to the vessel wall are critical to prevent stent migration. Chalouhi et al. [6] suggested using longer FDDs when there is a significant mismatch between the luminal diameters of the inflow and outflow vessels. Further, they also recommended avoidance of excessive dragging and stretching of the device to prevent the "accordion effect" [6]. When deploying an FDD, advancing, rather than unsheathing, the device and applying an intermittent forward force by pushing the wire and microcatheter will help the stent to be stable on the vessel wall in place. Lubicz et al. [7] suggest choosing a long length to ensure stability and use of a stent with diameters $0.25-0.50 \mathrm{~mm}$ larger than the distal parent artery diameter.

In the present case, migration could be explained by the "accordion effect." When deploying the stent on the dissecting segment, pushing the wire to create a stable apposition of the stent on the vessel wall was difficult because the diameter of the dissection was large. Therefore, we pulled the microcatheter to unsheathe it and deployed the stent. Since the dissecting segment was very long, microcatheter unsheathing was done over a long distance, and stretching of the stent seemed to occur which made foreshortening of the stent thereafter. And to avoid perforator occlusion we deployed the distal end of the stent not to cover BA as much as possible, so the coverage distal to the dissecting segment by the stent was too short, which was a mistake. The risk of occlusion of side branch artery is low (4.9\%) and most side-branch occlusion events have been reported to occur when more than two FDDs were used [8]. Therefore, we should have deployed the stent more distally in the BA, using two stents making the overlapping zone proximal to BA to avoid perforator occlusion, and to make a gen- erous stable coverage zone of the stent proximal and distal to the long unstable coverage zone in the dissecting segment.

In conclusion, migration of FDDs is usually silent, but it can be catastrophic due to hemodynamic changes caused by the device, resulting in aneurysm growth or rupture. We report a rare case of delayed migration of a PED during VADA treatment, which resulted in rupture of the aneurysm. Appropriate stable coverage of the lesion and FDD sizing are important in preventing this potential complication.

\section{CONFLICT OF INTEREST}

No potential conflict of interest relevant to this article was reported.

\section{REFERENCES}

1. El Ouadih Y, Coll G, Jean B, Coste A, Chaix R, Sakka L, et al. A rare complication of flow diverter: delayed migration causing aneurysm expansion and brainstem compression. Br J Neurosurg 2019 May 27 [Epub]. https:// doi.org/10.1080/02688697.2019.1617406.

2. Patel PD, Chalouhi N, Atallah E, Tjoumakaris S, Hasan D, Zarzour H, et al. Off-label uses of the Pipeline embolization device: a review of the literature. Neurosurg Focus 2017;42:E4.

3. Takong W, Kobkitsuksakul C. Delayed proximal flow diverting stent migration in a ruptured intracranial aneurysm: a case report. Neurointervention 2020;15:154-7.

4. Badruddin A, Lazzaro MA, Taqi MA, Zaidat OO. Downward migration of carotid stent on 8 months follow-up imaging: possible stent "watermelon- seeding" effect. J Neuroimaging 2011;21:395-8.

5. Navarro R, Cano EJ, Brasiliense LB, Dabus G, Hanel RA. Fatal outcome after delayed pipeline embolization device migration for the treatment of a giant superior cerebellar artery aneurysm: technical note for complication avoidance. Open J Mod Neurosurg 2014;4:163-8.

6. Chalouhi N, Tjoumakaris SI, Gonzalez LF, Hasan D, Pema PJ, Gould G, et al. Spontaneous delayed migration/shortening of the pipeline embolization device: report of 5 cases. AJNR Am J Neuroradiol 2013;34:2326-30.

7. Lubicz B, Collignon L, Raphaeli G, De Witte O. Pipeline flow-diverter stent for endovascular treatment of intracranial aneurysms: preliminary experience in 20 patients with 27 aneurysms. World Neurosurg 2011;76: 114-9.

8. Zhou G, Su M, Yin YL, Li MH. Complications associated with the use of flow-diverting devices for cerebral aneurysms: a systematic review and meta-analysis. Neurosurg Focus 2017;42:E17. 